\title{
Los modelos de educación multicultural e intercultural. Una revisión necesaria desde una sociedad diversa
}
» The models of multicultural and intercultural education. A necessary revision from a diverse society

\begin{tabular}{l} 
Celmira Castro Suárez \\
\hline Universidad del Atlántico \\
\hline
\end{tabular}

\section{Resumen}

El presente artículo hace una revisión a la producción teórica que se ha generado en torno a la educación multicultural e intercultural en las últimas décadas. Para ello nos basamos especialmente en la producción que sobre el particular se ha dado en España, país que ante la llegada de inmigrantes se ha visto en la necesidad de formular políticas que buscan hacerle frente a este fenómeno social desde la perspectiva educativa. Situación que para especialistas como Antonio Muñoz Sedano, Teresa Aguado, Marta Soriano se ha convertido en un importante objeto de investigación, al igual que ha ocurrido para el caso norteamericano con estudiosos como James Banks, quien considera que el modelo multicultural y el modelo holístico han sido claves a la hora de generar acciones de intervención en los espacios escolares caracterizados por la diversidad cultural.

Palabras clave: educación, multiculturalidad, interculturalidad, diversidad, inclusión, modelos educativos.

\begin{abstract}
This article reviews the theoretical production that has been generated around multicultural and intercultural education in recent decades. For this we rely especially on the production that has occurred in Spain in particular, a country that before the arrival of immigrants has been in need of formulating policies that seek to address this social phenomenon from the educational perspective. Situation that for specialists like Antonio Muñoz Sedano, Teresa Aguado, Marta Soriano has become an important object of investigation, as it has happened for the North American case with scholars like James Banks, who considers that the multicultural model and the holistic model have been key when generating intervention actions in school spaces characterized by cultural diversity.
\end{abstract}

Keywords: education, multiculturality, interculturality, diversity, inclusion, educational models.

\section{Cómo citar este articulo:}

Castro Suárez, C. (2019). Los modelos de educación multicultural e intercultural. Una revisión necesaria desde una sociedad diversa. Revista Amauta, 17(33), pp.87-104. http://dx.doi.org/10.15648/am.33.2019.7
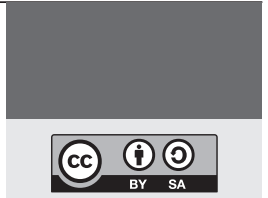

() 2019

Correspondencia de

autor:

celjosecastro@gmail.com

Recibido:

Agosto/5/2018

Aceptado:

Agosto/19/2018

Publicado:

3/Enero/2019 


\section{Introducción}

Al revisar la producción teórica sobre la interculturalidad, se observa que esta se sitúa entre los referentes de los grandes modelos de conocimiento surgidos en este campo o que han sido transplantados desde marcos más amplios. Es así como está presente el tecnológico, el hermenéutico y el crítico, los que desde perspectivas distintas proponen unas prácticas y unas estrategias para la acción educativa en contextos multiculturales (Yriarte, 2005, pp.69 y ss.).

Se observa también que cuando los especialistas hablan de modelo o paradigma intercultural, se refieren fundamentalmente a las diferentes maneras de aproximación a un ámbito de conocimientos determinados. Se muestra brevemente cada una de ellas:

Modelo tecnológico o positivista. Para este modelo, la resolución de los problemas planteados por la interculturalidad en el sistema educativo estriba en la adquisición de destrezas por los alumnos, que serían diseñadas por expertos educativos. Su orientación es de carácter fundamentalmente técnico y compensatorio, ya que el objetivo de la educación multicultural no es otro que el de compensar los déficits de las culturas distintas de la mayoritaria, tanto si esas carencias son reales 0 inventadas.

Modelo hermenéutico o interpretativo. Este sugiere que la educación intercultural debe nuclear su proyecto sobre la mejora del conocimiento de sí mismos, porque todos los alumnos deben de propiciarla, para estimular el desarrollo de la cooperación intercultural y reducir los prejuicios y discriminaciones. La perspectiva moral aparece en este modelo orientado a mejorar las habilidades de los sujetos para lograr una mejor autocomprensión desde la que se estimule la cooperación intergrupal.

Modelo crítico o sociopolítico. La propuesta de la educación multicultural se centra fundamentalmente en la modificación del currículum para adaptarlo a las exigencias planteadas por la democracia moral, que resultan incompatibles con la dominación cultural de unos grupos humanos sobre otros. Así entendida, la educación intercultural pretende que profesores y alumnos se comprometan en la modificación, tanto 
de las situaciones sociales como de los soportes ideológicos, que provocan la discriminación cultural y social (Soriano, 2002, p.84; Yriarte, 2005, p.70).

Tanto los anteriores modelos, como a los que posteriormente se hará referencia, son el reflejo de la influencia que sobre estos ejerció el movimiento multicultural, el cual como lo señala Muñoz Sedano

...es ante todo un fenómeno político y social de reivindicación de derechos humanos y civiles por parte de todos aquellos grupos que se sienten discriminados o marginados de la participación democrática ciudadana. Una lucha por la igualdad de oportunidades frente a los grupos culturales y sociales que ostentan el poder político y económico.

En efecto, estos modelos no pueden considerarse neutros, ya que en ellos está presente el compromiso o la postura ideológica de quien no solo estudia estos procesos sociales sino que además formula mecanismos, llámese modelos, enfoques o programas, de intervención social, lo que implica una determinada tendencia ideológica frente a la multiculturalidad.

Dentro de los especialistas que proponen otros modelos en relación con la educación multicultural, está Margarita Bartolomé (citada por Rodríguez Izquierdo, 2002, p.48) quien en su trabajo Educación multicultural: ¿En qué modelo nos situamos? Propone los siguientes modelos para tener en cuenta:

- Asimilacionista. No contempla las peculiaridades culturales de las minorías.

- Compensatorio. Parte de la idea de que las diferencias culturales se consideran un déficit que favorece el fracaso escolar.

- Currículo multicultural: Este introduce algunos contenidos "étnicos" en el currículo.

- Pluralismo cultural: Tiene como base el respeto, la diversidad y promueve la comunicación y el diálogo. 
En los planteamientos de Bartolomé está presente el modelo holístico propuesto por Banks, en el cual ya se incorporaban elementos de denuncia y lucha contra la discriminación y el racismo.

A continuación se describirán otros modelos existentes, con el interés de continuar el acercamiento a una primera revisión a la literatura que circula sobre el particular.

Modelo asimilacionista: Siguiendo con la descripción de los modelos y el análisis de estos enfoques, este modelo se enmarca dentro de la llamada "afirmación hegemónica de la cultura del país de acogida", es decir, se encuentra estrechamente relacionado con un modelo que pretende asimilar el inmigrante a la cultura, imaginarios y ritmos de la sociedad donde llega.

Este modelo asimilacionista, según Muñoz Sedano, "pretende la absorción de los diversos grupos étnicos en una sociedad que se supone relativamente homogénea, imponiendo la cultura del grupo dominante". Para ello se parte de la idea de que las sociedades desarrolladas son más universales y distan de los parroquianismos o particularismos, por lo que generan acciones de asimilación del individuo que llega, quien deberá asumir el rol de la cultura dominante. Con ello se pretende además debilitar los sentimientos étnicos, las divisiones, los separatismos y la "balcanización". En las sociedades de acogida la diversidad étnica, racial y cultural es vista como un problema que amenaza la integridad y cohesión social (Muñoz Sedano, p.3).

Este modelo tuvo un desarrollo histórico que se remonta a finales del siglo XIX y a la Primera Guerra Mundial. Pero fue en los años 60 donde tuvo un mayor auge, debido a la marcada inmigración que comenzó a vivir Europa y Estados Unidos En efecto, este concepto integracionista surgió en Estados Unidos a partir de la avalancha de los flujos poblacionales que llegaban al país. Este proceso de asimilación consistiría en unas acciones institucionales que desde arriba pretendían "simplificar" al individuo que llegaba al país, es decir, las minorías inmigrantes debían despojarse de todo aquello que les era distintivo, para copiar en sus formas de vida social y cultural a las mayorías de la sociedad receptora (Muñoz Sedano) En la práctica se trataba de un modelo de filosofía social y organización etnocultural orientado hacia la uniformidad u homogeinización de una sociedad, que al criterio de los políticos, "corría el riesgo de ser debilitada por los grupos étnicos y por los extranjeros que llegaban al país". 
La incidencia de este proceso es descrita para el caso inglés por Selby (citado por Rodríguez Izquierdo, 2002), quien por considerarlo ilustrador se trae a relación:

- La primera fase coincidió con la llegada al Reino Unido de los trabajadores del Caribe y Asia con sus familias en la década de los 50 y 60 . La palabra clave fue integración (o de acuerdo a algunos escritores, asimilación o absorción). El mundo educativo reaccionó a la presencia de hijos de inmigrantes en las escuelas suponiendo que ellos a un mismo tiempo, absorberían la "cultura dominante" y serían absorbidos por ella sin entrar en el significado de lo que entendían por tal proceso. (p.51)

Este proceso histórico vivido en Inglaterra nos lleva a reflexionar en torno a nuestra realidad social, la cual de cierta manera dista de las sociedades a las que pertenecen los teóricos aquí analizados. En efecto, en nuestros espacios educativos no es usual encontrar hijos de inmigrantes, pero sí se pueden hallarse personas de diferentes etnias o color, y sobre todo, también hay personas víctimas del desplazamiento forzado producto de la violencia que azota varios países de América Latina; caso particular el de Colombia, donde el sistema educativo debe empezar a replantear sus programas y contenidos curriculares, ya que estos deben tener en cuenta las necesidades de las personas que cada día se desplazan del campo a las grandes ciudades y tienen enormes dificultades para adaptarse a un mundo totalmente distinto que no les brinda las condiciones mínimas de vida.

Continuando con el modelo asimilacionista, el sociólogo de las migraciones Jordi Garreta llama la atención sobre la existencia de distintas tipologías en el proceso de asimilación social (Garreta Bochaca, 2003, pp.22 y ss.) las cuales reproducimos a continuación:

- La aculturación, que supone la interiorización de las culturas del grupo étnico dominante.

- La asimilación estructural, que supone la participación en organizaciones primarias del grupo étnico dominante con la separación de las propias.

- La amalgama o formación de parejas y matrimonios mixtos. 
- La asimilación identificacional o pérdida de la identidad para dar cabida a los sentimientos de pertenencia al grupo étnico dominante.

- La asimilación actitudinal, de comportamientos y valores que supondrían la ausencia de prejuicios, discriminación y conflictos.

Cada uno de estos planteamientos muestra el interés integracionista de un modelo que ha sido criticado por su fundamentación unívoca o de inmersión que pretende adecuar o modificar las conductas, los valores y los ritmos de vida de quienes llegan a determinados espacios socioculturales considerados como superiores (Rodríguez Izquierdo, pp.52 y ss.) Además estos tipos de asimilacionismo social, que pasan necesariamente por la asimilación lingüística, dejan ver claramente que seguimos siendo desde niños como unas esponjas, que absorbemos y nos dejamos absorber por el medio, entorno y cultura donde se haga necesario vivir y crecer.

Este modelo no está exento de cuestionamientos y dudas en cuanto a su validez y eficacia en los procesos de educación multicultural. En efecto, esta postura se generó inicialmente desde una idea neocolonial que considera al país que acoge como una "civilización superior", con una economía fuerte y con una cultura dominante que no puede arriesgarse ante la avalancha de extranjeros "culturalmente inferiores".

Los fundamentos de este modelo, unidos a situaciones que deben afrontarse en la realidad social donde pretende aplicarse, lo hacen fácilmente cuestionable. Circunstancia que generó debates académicos entre especialistas, quienes consideran que el mismo tiene problemas en cuanto a su aplicabilidad ya que, como lo exponen Banks y Linch, (citado en Rodríguez Izquierdo, 2002, p.53) se enfrenta con grandes problemas relacionados con el pluralismo étnico y cultural. Problemas que según estos teóricos, se explican de la siguiente manera:

- No hacía posible alcanzar atributos y conductas que se necesitan asimilar para lograr la plena integración.

- Internacionalización de los ideales de igualdad y democracia, creencias en que podían ser realizados. 
- No satisfacción de las demandas de empleo, participación política, justicia retributiva e incorporación de sus culturas y lenguajes en la cultura general del sistema escolar estatal.

- Fracaso de las naciones occidentales en el intento de disminuir las distancias entre sus ideales democráticos y las realidades sociales existentes en el entramado sociocultural.

Cualquiera que sea la propuesta educativa, basada en un modelo multicultural 0 etnocultural, debe ser planeada y pensada teniendo en cuenta el tipo de sociedad en la que se dan las situaciones, sin olvidar que cada fenómeno va mucho más allá de la escuela; es decir, se trata de reconocer la existencia de una realidad sociocultural en la cual se va a llevar a cabo el programa de intervención social.

En este sentido, se encuentra otro modelo:

Modelo estructural-funcional. Parte de la necesidad de tener en cuenta la interrelación entre todas las dimensiones y elementos del medio social y educativo (sistema educativo, centro escolar, grupos-clase)(Aguado, 1996, p.36) Aguado propone que el modelo psicológico debería estar presente en toda interpretación de las situaciones educativas multiculturales, al focalizarse en variables que modulan en gran medida los resultados educativos-motivación, autoconcepto.

En este orden de ideas, también plantea algunos factores que se deben tener en cuenta en todas las manifestaciones multiculturales, las cuales se presentan como conflictos sociales, agentes de cambio y recursos educativos, que se convirtieron en un reto por lograr en las sociedades occidentales. Estos son:

- La enorme distancia entre los ideales democráticos y las realidades actuales -minorías históricas, inmigrantes de sociedades despolarizadas y poco desarrolladas-. La internalización de los ideales de igualdad y democracia crea expectativas imposibles de alcanzar para los grupos minoritarios, lo cual genera presión sociopolítica. Los movimientos de revitalización étnica son un ejemplo de la identidad étnica al reclamar mejores condiciones de vida para grupos marginados (Féher, 1991) 
- Necesidad de aumentar los valores propios del país o grupo, y el universo de significados, o descubiertos estilos de aprender, motivaciones, valoración de las instituciones, socialización, etc. (Jordán, 1992, p.12)

- Independencia mundial en una sociedad global y cada vez más conectada en un sistema general de relaciones, a la vez que con una mayor tendencia a la uniformidad, con cierto sesgo hacia la mediocridad.

- La demanda de empleo, participación política, recursos económicos y la incorporación de sus culturas y lenguajes en la cultura general y en el sistema escolar, son reclamaciones legítimas en sociedades que se autocalifican de democráticas, y que proclaman tolerancia, respeto a la diferencia, participación, autonomía y libertad.

Teniendo en cuenta los anteriores factores, es necesario que toda propuesta educativa multicultural, pueda de alguna manera replantear los problemas que permitan dar respuesta a los problemas de equidad y desigualdad.

Modelo segregacionista. Surgió en Europa, como una política educativa, que consistía en segregar a los inmigrantes, y procuraba educarlos en su propia cultura y en su lengua de origen, para lo cual debían estar separados de los demás estudiantes y marcar la diferencia entre lo autóctono y lo no originario.

Es de anotar, que este modelo surgió paralelamente a los programas asimilacionistas que se desarrollaban en algunos países. Pretende que el Estado genere políticas de segregación para las minorías étnicas o grupos raciales determinados. Un ejemplo de ello, como lo anota Muñoz (p.4) fueron las reservas indias y las escuelas para negros. Prácticas que terminaron generando una serie de luchas por superar esta segregación en Estados Unidos (campaña por la igualdad de derechos civiles, en especial por la apertura de todas las escuelas a la población negra) y la lucha contra el appartheid en África del Sur.

Muñoz, comenta que en este modelo se aplicó también el llamado programa de diferencias genéticas, el cual partía de la idea malthusiana de que los alumnos de grupos étnicos minoritarios tenían peores resultados escolares a causa de sus características biológicas. Por lo tanto, se reagrupaba a los alumnos según su cociente 
intelectual o nivel, y se ofrecían programas distintos que conducían a carreras de mayor a menor prestigio. Este programa sería aplicado en varios países europeos y en Estados Unidos, generándose además una réplica que sería adaptada en países de América Latina, incluso al interior de las mismas escuelas.

Modelo compensatorio. Según Muñoz Sedano, este modelo parte de la idea de que los jóvenes pertenecientes a minorías étnicas crecen en contextos familiares y sociales en los que no gozan de posibilidades de adquisición de las habilidades cognitivas y culturales requeridas para funcionar con éxito en la escuela, por lo que necesitan ser recuperados de su déficit sociocultural mediante programas compensatorios 0 retributivos que les "faciliten" su inserción en la sociedad.

Este modelo considera al niño de un grupo minoritario como "culturalmente desvalido", por lo que de cierta manera se termina en la práctica asumiendo una actitud protectora y tutelar hacia el otro, al cual lo ve como desprotegido e indefenso desde el punto de vista cultural. De este modelo se derivan dos acciones o programas de educación: a) compensatorio de las desigualdades culturales y b) rechazo de sus raíces culturales. Con la primera se pretende equilibrar o brindar más oportunidades al joven estudiante proveniente de sectores marginados por lo que se le considera en inferiores condiciones. La segunda apunta a eliminar o despreciar las raíces culturales del inmigrante, o perteneciente a grupos minoritarios, para asimilarse así al grupo mayoritario o hegemónico. Se considera que si esto no se efectúa, se pueden generar conflictos al interior de la sociedad "culturalmente superior".

Para el caso español, Muñoz Sedano comenta que la educación de inmigrantes está siendo encomendada, en muchos lugares, a los programas y profesores de educación compensatoria o de atención a alumnos con necesidades especiales. Lo que lleva consigo un alto riesgo de aplicar las teorías y prácticas de educación compensatoria, ampliamente discutidas en la teoría y en la práctica. Es decir, a un desfase que resulta complejo.

Este tipo de modelo desarrolla programas destinados a gitanos y comunidades negras para el caso español. Ha sido criticado por algunos especialistas, ya que en la práctica terminan por "etiquetar al alumno de medio socioeconómico deprivado, 
como alumno deficiente, atribuyendo así su falta de rendimiento a una incapacidad debida al ambiente social y familiar" (Muñoz Sedano, p.13) También se analiza que

la adopción mental de esta postura teórica por parte del profesor hace bajar en éste las expectativas de un buen rendimiento; la comunicación consciente e inconsciente de estas bajas expectativas produce un efecto de baja estimulación y motivación en el alumno; con lo que se produce el nefasto resultado de la profecía que se cumple por sí misma: Efecto Pigmalión". (Muñoz Sedano)

Otro aspecto por el cual se critican los resultados obtenidos se relaciona con la errónea aplicación que de este modelo hacen algunos profesores que atienden estos sectores de la población marginada, quienes terminan por "atribuir el retraso escolar a una cultura y lengua originaria que hacen rendir menos [al estudiante] en el ambiente escolar, en el que sólo se utiliza la lengua y cultura dominante, más desarrollada y más avanzada" (Muñoz Sedano).

Modelo antirracista. Debido a la creciente xenofobia generada en países europeos, ejemplo España y Alemania, se viene repensando la llamada integración cultural, la cual parte del criterio de la interdependencia existente entre grupos portadores de diversas culturas, que tienen toda la capacidad de confrontar e intercambiar normas, valores, modelos de comportamientos, en el mismo nivel de igualdad y de participación.

En respuesta a esta xenofobia, que muchas veces refleja las mismas actitudes asumidas por los padres ante los hijos o por los medios de comunicación, se han generado algunos programas y prácticas educativas que apuntan a construir espacios contra el clima de intolerancia y racismo cultural. Es así como hoy se habla del "método para desarrollar la tolerancia" o "el método de cómo vivir juntos", los cuales se proponen "aprovechar la existencia de contextos heterogéneos para desarrollar la convivencia mediante el aprendizaje cooperativo" (Aguado, 1996, p.95). Con ello se pretende no solo de ocuparse para enseñar a los alumnos considerados en desventaja cultural y socioeconómica, sino enseñar de otra manera a todos los demás. 
El modelo antirracista apunta en definitiva a lograr el reconocimiento del pluralismo cultural, es decir, a valorar, respetar y reconocer la existencia de grupos culturales distintos dentro de la sociedad. Pero para que pueda darse esta integración cultural pluralista, según Muñoz Sedano

se requieren unas condiciones mínimas en la sociedad: reconocimiento explícito del derecho a la diferencia cultural; reconocimiento de las diversas culturas; relaciones e intercambios entre individuos, grupos e instituciones de las varias culturas; construcción de lenguajes comunes y normas compartidas que permitan intercambiar; establecimiento de fronteras entre códigos y normas comunes y especificas, mediante negociación; los grupos minoritarios necesitan adquirir los medios técnicos propios de la comunicación y negociación (lengua escrita, medios de difusión, asociación, reivindicaciones ante tribunales, manifestaciones públicas, participación en foros políticos...) para poder afirmarse como grupos culturales y resistir a la asimilación. (p.7)

Efectivamente, al no darse estas condiciones en la sociedad, al no darse una política integracionista que procure instaurarlas y desarrollarlas, difícilmente se logrará el éxito de este modelo. Pues de lo que se trata en la práctica educativa es de fomentar o hacer realidad una política integracionista capaz de crear una cultura común, que recoja las aportaciones de todos los grupos étnicos y culturales presentes en una sociedad.

Muñoz Sedano relata cómo esta tendencia por el aglutinamiento cultural

generó en los Estados Unidos el modelo de melting pot(crisol), sacado de una obra teatral estrenada en Nueva York en 1908, en la cual se concibe América como una nación en la que todas las diferencias étnicas se funden en una sola entidad nacional que es superior a todas ellas por separado. La pretensión es mantener la coexistencia y el equilibrio entre las culturas minoritarias y ofrecer lo mejor de la cultura dominante para todos. La teoría del melting pot se halla muy extendida en la sociedad estadounidense, como señala Bennet, y 
son en realidad muchos los educadores que consideran que su papel principal es conseguir que los grupos de niños y niñas procedentes de otros lugares del mundo se asimilen dentro de la cultura dominante.

Pero en la práctica esta tendencia integracionista es criticada por ser una posición "ambigua entre la idea progresista de la lucha por la igualdad de oportunidades y la teoría de la deficiencia, que acaba explicando los déficits de las minorías desde los propios estereotipos de estas" (Muñoz Sedano). Lo que en el espacio escolar se convierte en "una forma sutil de racismo y una creencia en la superioridad de la cultura receptora" (Muñoz Sedano).

Para Muñoz Sedano

el mito del "melting pot" ha resultado ser una falacia que camufla la ideología asimilacionista, puesto que la cultura anglosajona sigue siendo la dominante y el resto de grupos culturales tienen que renunciar a sus características étnicas para poder participar plenamente en las instituciones sociales, económicas y políticas de la nación.

Pero a pesar de sus aspectos negativos, hoy se reconoce que la educación no racista producto de la política educativa integracionista ha generado un acercamiento a la comprensión del mundo cultural al interior de los espacios escolares. Su pretensión apunta a construir un sentir positivo de unidad y tolerancia entre los estudiantes y dejar de lado los estereotipos que consideran que existen culturas superiores e inferiores.

El modelo no racista integra prácticas educativas que intentan reducir y eliminar actitudes xenófobas. Su centro de acción son los estudiantes y profesores del grupo cultural dominante para garantizar la convivencia racial, la comunicación y la tolerancia con estudiantes provenientes de sectores marginados 0 inmigrantes. Esto lo convierte en un modelo que confunde sus fronteras con el modelo intercultural. Pues en el espacio escolar lo que se persigue es la convivencia a través de la promoción y difusión del respeto y la aceptación intergrupal.

Para lograr esta meta se ha desarrollado un modelo pedagógico cuyos componentes básicos, Aguado (1996, p.97) los resume de la siguiente manera: 
- Aprendizaje cooperativo, mediante la formación de equipos de trabajo interétnico para fomentar la distribución del éxito académico, como condición básica para superar la discriminación.

- Discusión y representación de los conflictos originados por los perjuicios.

- Transformar la actividad escolar para acercarla a los alumnos en desventaja.

Según Aguado (1996) los resultados obtenidos, para el caso español, indican que se hace preciso intervenir en edades muy tempranas. Las diferencias étnicas y culturales son fuente de oportunidades educativas para desarrollar la tolerancia y la construcción de una identidad étnica positiva. El sistema resulta positivo para los alumnos aventajados, porque se sienten recompensados cuando se dan cuenta de que pueden enseñar a otros. Y por último, se reducen los prejuicios, aunque no aumenta la motivación para el aprendizaje ni cambia la relación con el profesor o el autoconcepto.

Modelo pluralista cultural. Este modelo surge como una manera de rechazar la jerarquización etnocéntrica de las culturas y de la afirmación de la diferencia cultural como positiva. En tal sentido, queda claro que estos dos planteamientos evolucionan hacia uno pluralista donde cada grupo cultural tiene el derecho a conservar y desarrollar su cultura, sus diferencias, tradiciones y sus propias escalas de valores. Este modelo conocido también como "interculturalismo" o "mosaico cultural", pretende la construcción de sociedades fuertemente cohesionadas, donde todos los individuos que las conforman interaccionan y participan igualmente, a la vez que mantienen sus propias identidades culturales.

Hay que tener en cuenta que este modelo es el resultado del pluralismo cultural, como ideología y como política, el cual propende por la defensa de todas y cada una de las culturas, así como de su conservación y al desarrollo de los espacios socioculturales en que habitan cada uno de estos grupos poseedores de una forma de vida colectiva que los caracteriza ante los demás. El significado de este modelo tiene que ver con su intención de fomentar el valor de la igualdad existente entre las culturas, lo que se explica en el sentido de "la convicción de que la existencia de cada cultura sólo puede asegurarse ratificando sus diferencias y particularidades con respecto a las 
demás"(Aguado, 1996). En la práctica este modelo también resultó ser una respuesta a las tendencias uniformadoras del asimilacionismo, que terminó por privilegiar la homogeneización cultural antes que la igualdad educativa.

Para este modelo, que sigue muy de cerca los postulados de Banks, la escuela debe ser el espacio natural en que se promuevan las identidades y el sentido de pertenencia étnica. Por ello, los programas escolares deben estar dirigidos a darle mayor preponderancia a las formas o estilos de aprendizaje de los grupos étnicos, así como a los contenidos culturales particulares. Ello implica que la práctica educativa debe abrir espacios para los estudios étnicos, ya sea incorporando estos temas al currículo o formando escuelas de carácter étnico propio, como catalizadoras y guardianes de las culturas y tradiciones.

Este modelo, con ciertos niveles de adaptación particular, ha logrado importantes avances en la educación multicultural para el caso latinoamericano. Prueba de ello resulta ser la llamada etnoeducación, la cual para Ecuador y Colombia (especialmente la zona del Cauca) ha permitido generar en los indígenas (considerados aún como minorías étnicas) una mayor conciencia de su identidad y el autorreconocimiento de sus propios valores culturales. Este proceso se ha visto fortalecido pero transformado a través de las prácticas educativas propias, que incluso llevaron a la institucionalización de la etnoeducación (Rojas \& Castillo, 2005, p.97)

Modelo multicultural y modelo holístico de Banks: Estos dos modelos educativos coinciden, pues a la larga pretenden fomentar la interculturalidad en los espacios educativos. Ambos parten de la concepción que la diversidad étnica y cultural resulta ser un elemento positivo y enriquecedor en la vida de toda persona, por lo que pretenden generar espacios de diálogo con y entre los estudiantes, para que se reconozcan y se acepten; así como también para reconocer al otro como diferente, pero en igualdad de derechos.

El primero de estos modelos apunta a la generación de competencias multiculturales en los estudiantes. Este es visto y definido por Banks (citado por Muñoz Sedano) como 
el proceso por el que una persona desarrolla un cierto número de competencias en múltiples sistemas de normas de percibir, evaluar, creer y hacer. Los individuos aprenden a movilizar, según las situaciones, competencias culturales diversas. Esto requiere una intensa interacción de individuos de culturas varias en la misma escuela(p.10)

El objetivo primordial tiene como norte "preparar a todos los alumnos -mayoritarios $y$, fundamentalmente, minoritarios- para poder comprender, adaptarse y funcionar adecuadamente, tanto en la cultura mayoritaria como en la minoritaria; es decir, generar una auténtica competencia multicultural". (Banks, citado por Muñoz Sedano) Este modelo tiene como principal característica su voluntad de facilitarle a cada uno de los estudiantes, cualquiera sea su origen o procedencia cultural, el análisis de los conceptos y temas desde distintos ángulos u orillas culturales, para fomentar en estas aptitudes y actitudes de empatía y valoración de las diferencias, según Sabariego (2002, p.23). Esto implica desarrollar en la comunidad estudiantil, a través del currículo, conocimientos, habilidades y actitudes, así como cualidades, para poder construir un diálogo fluido entre las culturas, independientemente de la orilla o frontera cultural desde donde se esté situado.

Banks (1991, p.130) incorpora a este modelo unos alcances operativos que sugieren un contexto más amplio desde la perspectiva curricular, lo cual significa la necesidad de vincular toda la actividad escolar en este proceso, a la par que se fomenta el análisis crítico y la reflexión sobre la realidad social por parte de todos los actores que conforman el escenario escolar. Construye así un puente integrador entre el enfoque intercultural y el sociocrítico.

Muñoz Sedano, citando a Banks, da cuenta de cómo

el modelo holístico de Banks supone la creación de un ambiente escolar definido por los siguientes rasgos: el personal de la escuela tiene valores y actitudes democráticas (no racistas); la escuela tiene normas y valores que reflejan y legitiman la diversidad cultural y étnica; los procedimientos de valoración y evaluación promueven la igualdad étnica y de clase social; el currículo y los materiales de enseñanza presentan perspectivas diversas étnicas y culturales en concepto, 
aplicaciones y problemas; el pluralismo lingüístico y la diversidad son valorados y formulados en la escuela; se utilizan maneras de enseñar y estilos de motivación que son efectivos con grupos de estudiantes de diferente clase social, raza o etnia; profesores y estudiantes adquieren las habilidades y perspectivas necesarias para reconocer las diversas formas de racismo y desarrollar acciones para eliminarlo. (p.17)

Al respecto, Banks insiste en la necesidad de fomentar la dimensión crítica del currículo, ya que este

debe ayudar a los estudiantes a desarrollar el conocimiento y habilidades necesarias para examinar críticamente la estructura política y económica actuales, así como los mitos e ideologías usados para justificarlas. También un currículo debe enseñar a los estudiantes las habilidades de pensamiento crítico, los modos de construcción del conocimiento, las asunciones básicas y los valores que subyacen a los sistemas de conocimiento y cómo construyen el conocimiento ellos mismos". (Banks, 1991, p.130)

En los planteamientos de Banks, en torno al papel que debe jugar la educación intercultural, están implícitos dos principios claves: a) Uno, a través del cual propone que todo plan de estudios debe convertirse en una herramienta para ayudar a los estudiantes a entender cómo todo conocimiento se construye socialmente, al mismo tiempo que este termina por ser el reflejo de las experiencias personales de los investigadores, así como los contextos sociales, políticos, y económicos en los que estos viven y trabajan. (Banks) b) La otra parte de la idea: que la escuela debe proporcionarles oportunidades de participación a todos los estudiantes en las actividades curriculares desarrolladas, lo cual permite que el conocimiento, las habilidades y las actitudes desarrolladas aumenten los logros académicos y que las relaciones de carácter interracial sean mucho más positivas (Banks).

En todo caso, es importante anotar, que existen diversos modelos y muchas teorías en relación con el tema de la educación multicultural, a los cuales se hace necesario tener en cuenta a la hora de construir los referentes teóricos y conceptuales de trabajos de investigación que se ocupen de la educación en sociedades diversas como la del Caribe colombiano. 


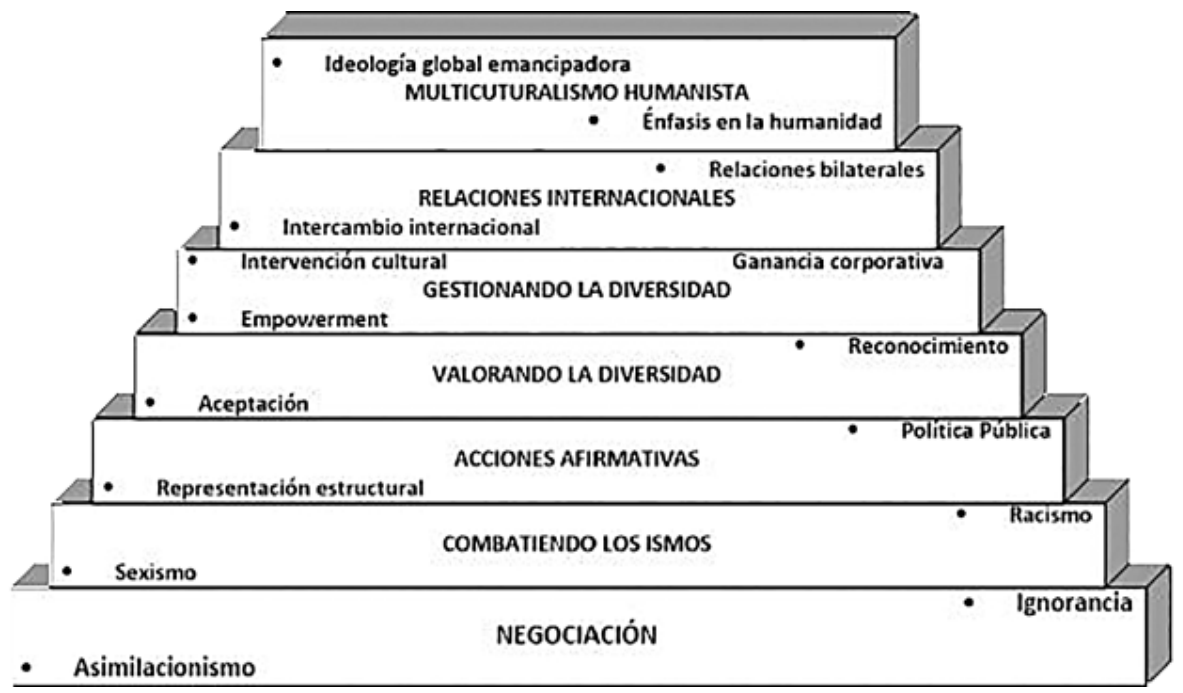

Figura 1. Piramidal de los modelos de Multiculturalismo

Fuente: Tomado de Sabariego, M. (2002). La Educación Intercultural ante los retos del siglo XXI. Bilbao, Descleé, p.96 

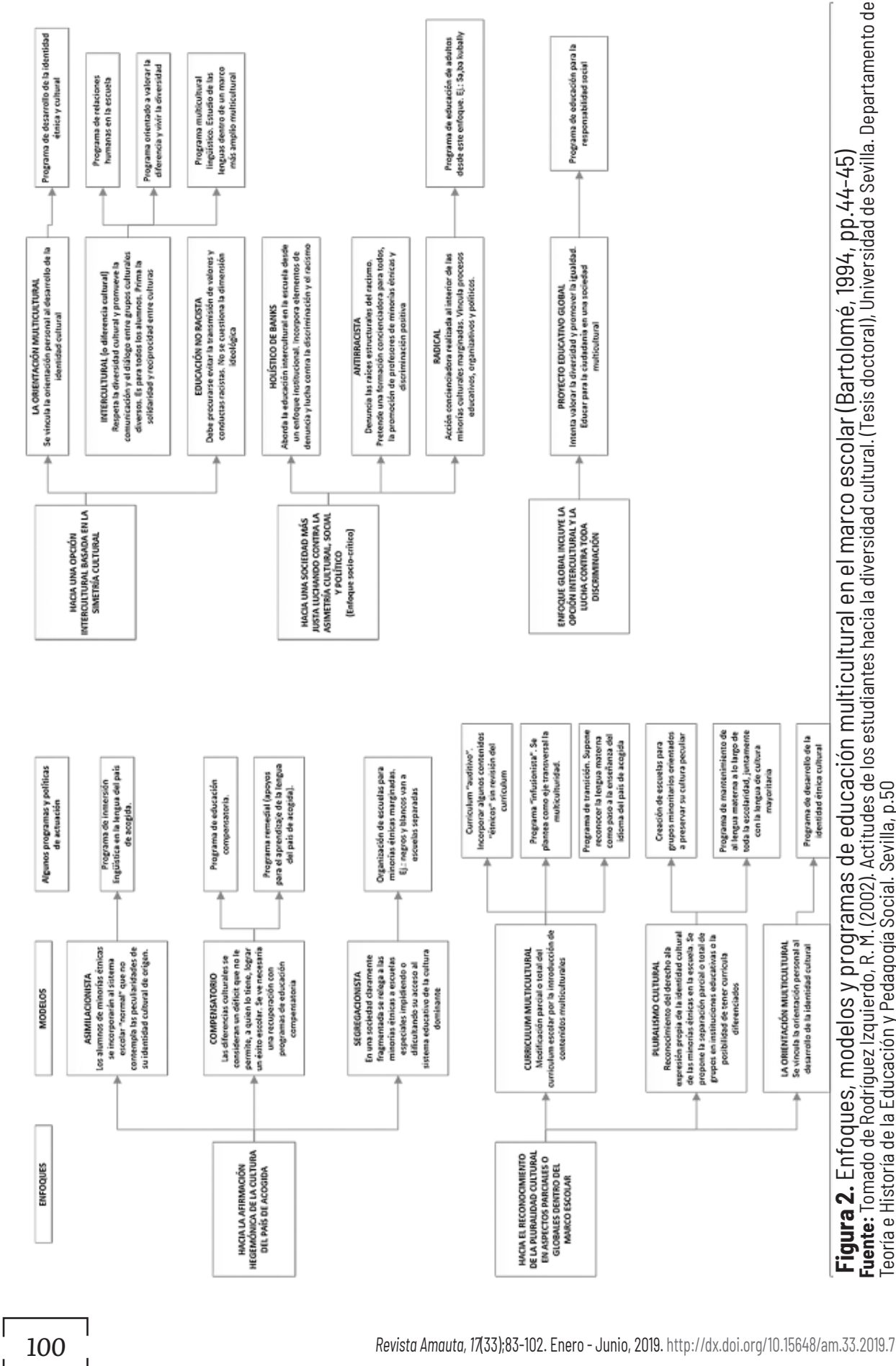

Revista Amauta, 17(33);83-102. Enero - Junio, 2019. http://dx.doi.org/10.15648/am.33.2019.7 


\section{REFERENCIAS BIBLIOGRÁFICAS}

Aguado, M. T. (1996). Educación multicultural. Su teoría y su práctica. Madrid: UNED.

Banks, J. Diversity within unity: Essential principles for teaching and learning in a multicultural society by http://www.nsrfharmony.org/pcc-equity/diversity_unity. pdf (Consultado 22 de mayo de 2008).

Banks, J. (1991). Teaching multicultural literacy to teachers. Teaching Educativa, 4(1), 130.

Garrieta Bochaca, J. (2003). La integración sociocultural de las minorías étnicas: gitanos e inmigrantes. Barcelona: Anthropos.

Jordán, J. (1992). La educación multicultural. Barcelona: CE-AC.

Muñoz Sedano, A. Enfoques y modelos de educación multicultural e intercultural. Recuperado de: www.madrid.org/webdgpe/interculturalidad/enfoques.doc (Consultado 29 de marzo de 2007).

Rodríguez Izquierdo, R. M. (2002). Actitudes de los estudiantes hacia la diveridad cultural. (Tesis sin publicar). Universidad de Sevilla, España.

Rojas, A. \& Castillo, E. (2005). Educar a los otros. Tunja: Universidad del Cauca.

Sabarriego, M. (2002). La educación interculturalidad ante los retos del siglo XXI. Bilbao: Desclée de Browe.

Soriano, M. (2002). La educación intercultural ante los retos del siglo XXI. Bilbao: Desclée.

Yriarte, R. M. (2005). Pluralidad y educación. La intercultura como modelo educativo. En: T. Fernández García et al., Multiculturalidad y educación. Teoria, ámbitos y prácticas. Madrid: Alianza. 